\title{
Assessment of Knowledge of Medical Students on an Important Health Issue Neagleria Flowri Infection at a Medical College of Karachi, Pakistan
}

\author{
Syed Ijlal Ahmed ${ }^{1, *}$, Syeda Beenish Bareeqa ${ }^{2}$, Munazza Suherwardy Obaid $^{3}$, \\ Saad Qureshi', Samra Khan', Sara Memon ${ }^{2}$ \\ ${ }^{1}$ Liaquat National Medical College and Hospital, Pakistan \\ ${ }^{2}$ Jinnah Medical and Dental College, Pakistan \\ ${ }^{3}$ Department of Public Health, Dow University of Health Sciences, Pakistan
}

Copyright $(2017$ by authors, all rights reserved. Authors agree that this article remains permanently open access under the terms of the Creative Commons Attribution License 4.0 International License

\begin{abstract}
Background: Since 2005 Neagleria Flowri infection appeared as a major health issue in Karachi, Pakistan. Most infections were acquired due to swimming in fresh water, ablution with fresh stagnant water. Our objective was to assess the knowledge of medical students regarding this important health issue and to highlight any deficiencies in medical education system. Material and methods: It was a prospective cross sectional study conducted at a medical college of Karachi, Pakistan. The sample size was calculated and sampling technique was randomized stratified sampling. Knowledge of the students was assessed through designed questionnaire. The data was recorded and analyzed on SPSS vs 21. Results: Our study included 250 medical students from five years of medical school. The highest response was recorded from final year regarding type of organism and habitat. Regarding treatment of Naegleria most correct response was from 4th year MBBS. Most final years answered correctly regarding the ablution as risk factor. The knowledge of medical students regarding incubation period of Neagleria was generally low. Conclusion: The knowledge of medical students regarding this significant medical issue appears low. The response of medical students was generally poor regarding treatment of infection, incubation period of infection and earliest presenting symptom of infection. Important measures should be taken to improve the knowledge of medical students regarding this significant health issue.
\end{abstract}

Keywords Neagleria, Ablution, Medical Students

\section{Introduction}

Naegleria Flowri, a pathogenic and opportunistic free-living amoebae, is an aerobic, mitochondriate, eukaryotic protozoa that occurs world-wide and causes an acute, fulminant, necrotizing and hemorrhagic meningoencephalitis called Primary Ameobic Meningeoencephalitis (PAM)* that may be fatal in immune-competent individuals with a history of recent contact with fresh water [1].

Infections can occur when N. Flowri enters the brain via the cribriform plate by travelling through the olfactory nerve tissue as water contaminated with N. Flowri is taken in through the nose [2]. Although majority of the cases have occurred in the United States, infections have been documented in countries throughout the world. Previously cases of PAM were reported from Europe, especially from the Czech Republic, Belgium, and the United Kingdom. The first case of PAM in Italy, was diagnosed through a postmortem in a healthy child who had possibly acquired the infection after swimming in puddle of stagnant river water [3]. Only 3 out of the 133 patients with PAM have been successfully treated in the United States from 1962 to 2014 [4].

Aggressive treatment with intravenous and intrathecal Amphotericin B and Miconazole along with oral rifampin had proved to be successful in a girl from California, who was followed over a period of 4 years and remained free of any neurological manifestations of the disease [1]. None of the PAM cases reported in Pakistan so far has survived.

In Pakistan PAM was first reported in 2005 [5]. Since then, there has been a rise in the incidence in Karachi, Pakistan. This increase in the incidence of PAM has been associated with swimming in freshwater and inadequately chlorinated swimming pools. Routinely performed ritual ablution by Muslims has also been associated with the outbreak in this part of the world [6]. The hot and dry climate of Karachi favors the growth of the organism. 
According to the best our knowledge much less information is available regarding awareness in medical students regarding the significant health issue i.e. Neagleria Flowri infection.

The objective of our study is to evaluate the awareness of Neagleria Flowri infection among medical students which is a significant health issue in Pakistan. It will help to design public health strategies and awareness programs which will help to prevent the infection.

\section{Material and Methods}

Our study was a prospective comparative cross sectional study which was conducted from $1^{\text {st }}$ march to $30^{\text {th }}$ march 2016 at Liaquat National Medical College and hospital, Karachi, Pakistan.

The sample size was calculated by WHO sample size calculator. The sampling technique of our study was stratified randomized sampling. All five batches were evaluated through questionnaire based survey. The participants were divided into male and female.

The data was collected by trained medical students through questionnaire based survey. Data was record and analyzed on IBM statistics SPSS vs 21 .

The inclusion criterion of our study was all healthy medical students of five batches who willingly participated in the study.

The exclusion criterion was exclusion of faculty of medical college and staff.

The variables of our study included age of participants, batch of participants, gender of participants, organism type, disease caused by Naegleira Flowri, route of transmission of infection, person to person transmission, treatment of Neagleria infection, habitat of Neagleria, incubation period of infection, earliest symptom of infection, ablution as risk factor.

The ethical considerations were taken during study.

\section{Results}

Our study included 250 medical students from five years of medical school. The $40 \%$ were males and $60 \%$ were females. The responses to the questions regarding Neagleria Flowri infection are shown in table 1.

The highest correct response regarding the type of organism was from final year MBBS which was 44(91.7\%). Regarding disease caused by Neagleria Flowri highest correct response was also from final year MBBS which was 45(93.8\%). The route of transmission was most correctly answered by final year which was $47(97.5 \%)$. Regarding the treatment of Naegleria highest correct response was from fourth year MBBS i.e. 26(53.1\%).

Regarding the habitat of Neagleria final year answered as highest correct responses which were 35(72.9\%). Surprisingly the most correct responses regarding incubation period were from third year MBBS i.e. 12(27.9\%). First year MBBS has given most correct responses regarding earliest symptom which were $34(72.3 \%)$.

Regarding ablution as a risk factor most correct responses were from final year i.e. 38 (79.2\%). Regarding prevention of negleria infection by chlorination, final year MBBS given the most correct responses i.e. 38(84.4\%). Regarding diagnostic tests most correct responses were from fourth year MBBS i.e. 47 (94\%).

Table 1. Frequencies and percentages of students who answered correctly

\begin{tabular}{|c|c|c|c|c|c|c|c|c|c|c|}
\hline \multicolumn{11}{|c|}{ FREQUENCIES AND PERCENTAGES OF STUDENTS WHO ANSWERED CORRECTLY } \\
\hline $\begin{array}{c}\text { NIGLERIA } \\
\text { TYPE OF ORGANISM }\end{array}$ & \multicolumn{2}{|c|}{$\begin{array}{c}\text { FIRST YEAR } \\
\text { MBBS }\end{array}$} & \multicolumn{2}{|c|}{$\begin{array}{c}\text { SECOND YEAR } \\
\text { MBBS }\end{array}$} & \multicolumn{2}{|c|}{$\begin{array}{c}\text { THIRD YEAR } \\
\text { MBBS }\end{array}$} & \multicolumn{2}{|c|}{$\begin{array}{c}\text { FOURTH YEAR } \\
\text { MBBS }\end{array}$} & \multicolumn{2}{|c|}{$\begin{array}{c}\text { FINAL YEAR } \\
\text { MBBS } \\
\end{array}$} \\
\hline DISEASE & 31 & $62 \%$ & 28 & $60.9 \%$ & 26 & $60.5 \%$ & 45 & $90 \%$ & 45 & $93.8 \%$ \\
\hline $\begin{array}{c}\text { ROUTE OF } \\
\text { TRANSMISSION }\end{array}$ & 16 & $32 \%$ & 29 & $59.2 \%$ & 28 & $65.1 \%$ & 43 & $86 \%$ & 47 & $97.9 \%$ \\
\hline TREATMENT & 7 & $14 \%$ & 22 & $44.9 \%$ & 10 & $23.3 \%$ & 26 & $53.1 \%$ & 25 & $52.1 \%$ \\
\hline HABITAT & 22 & $44 \%$ & 22 & $44 \%$ & 24 & $55.8 \%$ & 25 & $50 \%$ & 35 & $72.9 \%$ \\
\hline INCUBATION PERIOD & 8 & $17 \%$ & 8 & $16.7 \%$ & 12 & $27.9 \%$ & 9 & $18 \%$ & 10 & $20.8 \%$ \\
\hline EARLIEST SYMPTOM & 34 & $72.3 \%$ & 34 & $70.8 \%$ & 21 & $48.8 \%$ & 36 & $72 \%$ & 28 & $59.6 \%$ \\
\hline $\begin{array}{c}\text { ABULITION AS RISK } \\
\text { FACTOR }\end{array}$ & 17 & $36.2 \%$ & 31 & $64.6 \%$ & 20 & $46.5 \%$ & 34 & $68 \%$ & 38 & $79.2 \%$ \\
\hline DIANOSTIC TEST & 18 & $38.3 \%$ & 27 & $56.3 \%$ & 21 & $48.8 \%$ & 47 & $94 \%$ & 44 & $91.7 \%$ \\
\hline
\end{tabular}




\section{Discussion}

Neaglaria Flowri is a rare pathogen and the primary causative agent of Primary Amoebic Meninogencephalitis. It is a thermophilic organism mostly inhabitant of fresh water lakes, hot springs, rivers, ponds and non-chlorinated waters [6]. First discovered in 1989 this organism infects the central nervous system primary and hence commonly known as the brain eating amoeba [7]. Despite the scarcity of this pathogen the case fatality is more than $95 \%$ of the cases affected despite advances in antimicrobial therapies [8]. A lot of research has taken place on this organism regarding the pathogenesis of this organism and many risk factors have been identified. In this study we assess the knowledge of medical students both in clinical and non-clinical years regarding their awareness of Neaglaria Flowri regarding its disease, mode of spread and risk factors.

Medical students being the primary respondents of this cross-sectional study were assessed. As it is shown in the study that most of the respondents knew that Neaglaeria is an amoeba and it is the causative agent of Primary Amoebic meningoencephalitis. It is well established that the organism enters the brain through the cribriform plate first by entering the nose and further research reveals that Neaglaeria infection hence cannot be caused by drinking water [9]. Knowledge assessed among final year medical students 97.9\% was much higher than the students of first year MBBS which was $32 \%$ regarding its mode of transmission being primarily through the nasal route.

Diagnosis of Neaglaria Flowri is established via clinical signs and symptoms and presence of trophoblasts in the CSF [1], regarding diagnosis $91.7 \%$ among final year students while among first year it was only $38.3 \%$. Clinical signs and symptoms of the infections may present as early as 24 hours or up to 2-8 days of infectivity. The most common signs and symptoms are those of meningitis including severe fever, chills, positive Brudzinski and Kernig signs along with photophobia, confusion, seizures, and possibility of coma [10]. On assessment of medical students regarding the incubation period we found much less knowledge overall i.e $20.8 \%$ in final year which was maximum. Regarding earliest symptom of disease surprisingly the lowest knowledge was among third year medical students which show the area of improvement.

Neaglaria Flowri has been associated with various factors. We have outlined above potential sources of contamination of this pathogen. Swimming has been associated as a major risk factor of possible infection. Majority of the studies from Pakistan reported infectivity despite the uses of water recreational activities. One possible cause could be attributed due to ritual of ablution. This is still debatable as outlined in a review by Fatima Mukhtar et as "Why males? Why young males? What predisposes males as compared to females for developing PAM? Why the younger age group is most affected? [4].
Ablution which is also known as "wudu" is common holy practice among Muslims all around the world. Cleaning nose with tap water is one of the significant components of ablution. It exposes the individuals to Negleria infection in endemic areas. We suggest using boiled or chlorinated water for this component of ablution. Regarding knowledge of ablution as risk factors, students shown good knowledge from $36.3 \%$ in first year to final year with $79.2 \%$.

Most of our students agreed that infection can be caused by swimming in contaminated waters.so that should be avoided.

Regarding chlorination of water as preventive measures the most of the responses of students were correct. The pools should be chlorinated in endemic areas.

\section{Conclusions}

The knowledge of medical students regarding this significant medical issue appears low. The response of medical students was generally poor regarding treatment of infection, incubation period of infection and earliest presenting symptom of infection. Important measures should be taken to improve the knowledge of medical students regarding this significant health issue.

\section{REFERENCES}

[1] Visvesvara, G.S., H. Moura, and F.L. Schuster, Pathogenic and opportunistic free-living amoebae: Acanthamoeba spp., Balamuthia mandrillaris, Naegleria fowleri, and Sappinia diploidea. FEMS Immunology \& Medical Microbiology, 2007. 50(1): p. 1-26.

[2] Baig, A.M., Pathogenesis of amoebic encephalitis: Are the amoebae being credited to an 'inside job' done by the host immune response? Acta tropica.2015 148: p. 72-76.

[3] Cogo, P.E., et al., Fatal Naegleria fowleri meningoencephalitis, Italy. Emerg. Infect Dis, 2004. 10(10): p. 1835-7.

[4] Mukhtar, F. and M.S. Wazir, NAEGLARIA FOWLERI: THE BRAIN EATING AMOEBA OR AN ENIGMA? Journal of Ayub Medical College Abbottabad.2015 27(3): p. 735-736.

[5] Ul Islam, M.Y., S.A. Rahim, and A. Salim, The dangerous turn of "brain eating amoeba" in Sindh, Pakistan. Journal of infection and public health.2015 8(3): p. 305-306.

[6] Shakoor, S., et al., Primary amebic meningoencephalitis caused by Naegleria fowleri, Karachi, Pakistan. Emerging infectious diseases.2011 17(2): p. 258.

[7] Marciano-Cabral, F. and G.A. Cabral, The immune response to Naegleria fowleri amebae and pathogenesis of infection. FEMS Immunology \& Medical Microbiology, 2007. 51(2): p. 243-259. 
[8] Diaz, J., Seasonal primary amebic meningoencephalitis (PAM) in the south: summertime is PAM time. The Journal of the Louisiana State Medical Society: official organ of the Louisiana State Medical Society.2011 164(3): p. 148-50, 152-5.

[9] Edagawa, A., et al., Isolation and genotyping of potentially pathogenic Acanthamoeba and Naegleria species from tap-water sources in Osaka, Japan. Parasitology research, 2009. 105(4): p. 1109-1117.

[10] Grace, E., S. Asbill, and K. Virga, Naegleria fowleri: pathogenesis, diagnosis, and treatment options. Antimicrobial agents and chemotherapy.2015 59(11): p. 6677-6681. 